Ann. Sci. Forest., 1977, 34 (3), 215-229.

\title{
Index foliaire, paramètres foliaires et caractéristiques édaphiques stationnelles dans quelques peuplements forestiers de Lorraine belge
}

\author{
J. P. VANSEVEREN *, et J. HERBAUTS ** \\ * Université Libre de Bruxelles. \\ Laboratoire de Botanique systématique ef d'Ecologie. \\ 28 av. P. Héger, 1050 Bruxelles \\ ** Fondation Universitaire Luxembourgeoise, \\ rue des Déportés, 140, 6700 Arlon
}

\section{Résumé}

\begin{abstract}
Dans onze peuplements feuillus de Lorraine belge, principalement des hêtraies, croissant sur une gamme variée de sols, nous avons comparé, au cours d'une même année, un ensemble de caractéristiques intrinsèques des peuplements (paramètres foliaires et index foliaire) aux facteurs édaphiques stationnels (type de sol, type d'humus, taux de saturation des horizons humifères, rapport $C / N$, disponibilité azotée potentielle, etc.). Des corrélations significatives ont pu être décelées entre certains facteurs pédologiques (notamment le rapport $\mathrm{C} / \mathrm{N}$ et la qualité minéralisatrice des humus) et la surface spécifique des feuilles de hêtre. Il est apparu que la surface spécifique des feuilles de Fagus a tendance à augmenter à mesure que diminue la qualité édaphique des stations.
\end{abstract}

\section{I. - Introduction}

L'action des facteurs du milieu et particulièrement des caractéristiques pédologiques sur le comportement et la productivité des essences forestières en région tempérée a déjà fait l'objet de tant de travaux qu'il peut paraître vain de vouloir encore aborder le sujet. Dans un article de synthèse paru en 1970, Garbaye et al. ont d'ailleurs fait une mise au point très complète sur les méthodes d'étude des relations milieuproduction qui, en recherche forestière, se sont développées et perfectionnées au fil du temps. Ces mêmes auteurs, se basant sur de nombreux travaux effectués sur ce thème depuis une cinquantaine d'années, ont proposé le canevas d'une méthode de travail permettant d'aboutir à une meilleure connaissance des relations liant facteurs du milieu et caractéristiques des peuplements.

Par le biais de recherches complémentaires effectuées dans une même région, l'occasion nous est fournie de pouvoir confronter, dans l'optique proposée par les auteurs précités, d'une part un ensemble de données relatives à des caractéristiques intrinsèques de peuplements feuillus (paramètres foliaires ef index foliaire), d'autre part des caractéristiques du milieu sous forme de variables édaphiques, soit synthé- 
tiques (type génétique de sol, type d'humus), soit sélectives (notamment la capacité minéralisatrice des horizons humifères), et ceci pour une gamme fort variée de types forestiers. II nous a, dès lors, paru utile de faire état des résultats obtenus.

Le nombre de stations pour lesquelles nous disposons de ce couple de données reste néanmoins modeste (11 stations), essentiellement parce qu'il s'avère difficile, sur le plan pratique, d'augmenter sensiblement l'échantillonnage sans alourdir considérablement le travai! de terrain. II a toutefois été possible de déceler des corrélations statistiquement valables dans les limites de cet échantillonnage.

\section{II. - Choix et description des stations}

Les 11 sites forestiers sélectionnés pour la réalisation de cette étude sont situés dans le sud-est de la Belgique (Lorraine belge) (voir croquis de situation de la fig. 1).

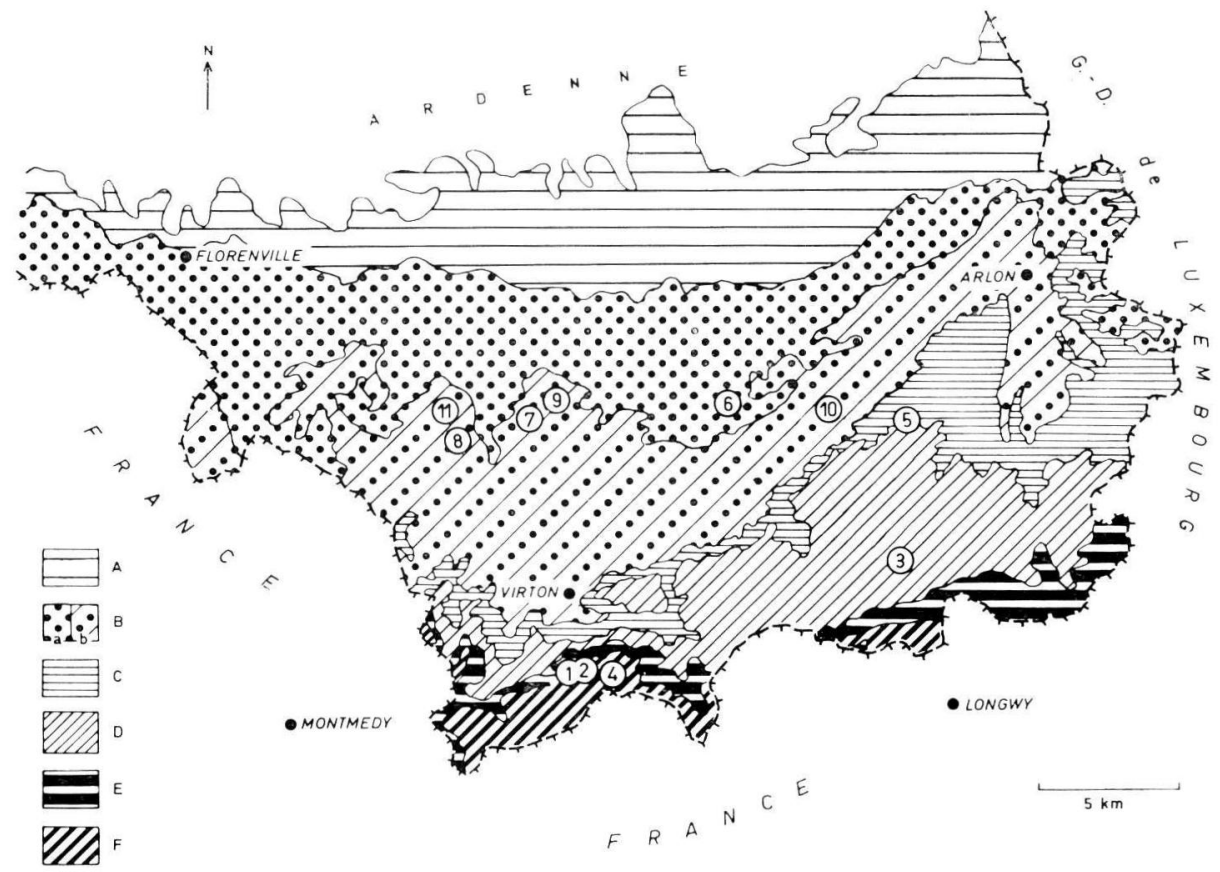

FIG. 1. - Croquis de situation et localisation des stations étudiées dans le cadre phyto-pédologique régional.

Geographical localisation of the studied forest stands in the pedological environment of the country.

A. chênaies-charmaies, sols bruns et bruns lessivés marmorisés sur marnes triasiques ; B. hêtraieschênaies acidophytes, sols bruns acides à podzoliques sur sables d'altération des grès calcaires infraliasiques $(a=$ Sinémurien, $b=$ Lotharingien) ; $C$. chênaies-charmaies, sols bruns et bruns lessivés marmorisés, sur marnes carixiennes ; D. hêtraies-chênaies mésophytes, sols bruns et bruns lessivés sur grès argileux domériens ; $E$. chênaies-charmaies neutrophytes, sols bruns eutrophes marmorisés sur marnes toarciennes ; F. hêtraies mélangées calcicoles, sols bruns calciques et rendzines brunifiées sur calcaires bajociens et hêtraies mélangées mésophytes sur loess et terra fusca. 
La diversité lithologique des sédiments jurassiques régionaux y est telle que la gamme des sols rencontrés, et donc des types forestiers qui y sont liés, est elle-même fort large : elle s'étend des rendzines brunifiées sur calcaires bajociens aux sols ocres podzoliques sur sable d'altération des grès quartzeux à ciment calcaire du Lias inférieur. Les associations forestières qui se partagent ce petit terroir sont le reflet de cette diversité édaphique, en sorte que la palette écologique des types forestiers est extrêmement diversifiée et se prête donc parfaitement à une sélection de stations contrastées dans un terriroire de superficie restreinte.

Du point de vue climatique la dition est influencée par un gradient climatique nord-sud qui affecte sensiblement le montant des précipitations et les valeurs des températures annuelles moyennes depuis le piémont du massif ardennais, au nord, jusqu'à la côte bajocienne, au sud, où s'affirme une tendance semi-continentale plus caractéristique du climat lorrain proprement dit (les extrêmes climatiques sont les suivants : $P=1100$ millimètres et $t m=7,5^{\circ} \mathrm{C}$ dans le bassin de la Semois; $P=900$ millimètres et $\mathrm{tm}=9^{\circ} \mathrm{C}$ sur la cuesta bajocienne). Toutes nos stations sont toutefois situées au sud du front de côte infraliasique, c'est-à-dire au sud du bassin de la Semois, en sorte que nous pensons pouvoir considérer l'influence du gradient climatique comme modérée, sinon négligeable pour le type de mesure qui fait l'objet de la présente note.

La composition des associations forestières est d'ailleurs, dans ce contexte régional, bien davantage inféodée au canevas lithologique : hêtraie mélangée calcicole sur les calcaires bajociens, hêtraies-chênaies mésophiles à flore du mull sur les grès médioliasiques et les placages loessiques, chênaies-charmaies mésophiles ou eutrophes sur les argiles carixiennes et les marnes toarciennes, hêtraies-chênaies acidoclines à flore du mull acide et du moder et hêtraies, hêtraies-chênaies ou chênaies acidophytes à flore du moder et du moder-mor sur les sables quartzeux résultant de la décarbonatation des grès calcaires sinémuriens ef lotharingiens (Duvigneaud 1960, Tanghe 1967, Sougnez 1967, Coûteaux 1969, etc.). Le gradient climatique sus-mentionné ne vient apporter que quelques retouches floristiques à ce schéma général essentiellement édaphique.

C'est au sein de cette mosaïque forestière qu'ont été sélectionnées 11 stations qui sont l'image assez fidèle des principaux types forestiers régionaux, à l'exclusion toutefois des chênaies-charmaies des sols argilo-marneux.

Les stations de Saint-Mard I et Saint-Mard II sont représentatives des hêtraies calcicoles (CariciFagetum) sur rendzines brunifiées (Saint-Mard I) et des hêtraies mélangées mésophiles (Melico-Fagetum) sur limon et terra fusca (Saint-Mard II) de la côte des calcaires bajociens ; la station de Saint-Mard III, plus particulière, représente un faciès d'acidification lié à l'existence de placages loessiques désaturés recouvrant, sur le revers de cette même cuesta, d'anciens matériaux siliceux de remplissage karstique.

Les sites de Musson et d'Udange sont caractéristiques des très productives hêtraies-chênaies mésophiles, à mélique et aspérule (Melico-Fagetum), parfois acidoclines, de la zone d'affleurement des grès argilo-ferrugineux domériens.

Les hêtraies et chênaies-hêtraies à flore acidophyte (Luzulo-Fagetum et Luzulo-Quercetum) qui s'étendent largement sur les substrats sableux acides issus de la décarbonatation des grès calcaires infraliasiques, sont reprises dans notre échantillonnage par 5 stations, selon une séquence croissante de podzolisation et d'acidification corrélative des humus : hêtraie à flore du mull acide sur sol brun acide légèrement cryptopodzolique (station d'Etalle), hêtraies à flore du moder sur sols bruns cryptopodzoliques (stations de Meix-devant-Virton et de Gérouville), chênaies-hêtraies à flore du moder ou du moder-mor, sur sols ocres podzoliques (stations de Sainte-Marie et de Bellefontaine). 
La liste ci-dessous regroupe les 11 stations retenues. Le classement correspond à un gradient d'humus de moins en moins actifs. Sont mentionnés succinctement : le type forestier défini par les essences dominantes de la strate arborescente et les groupes écologiques constituant la strate herbacée, la situation topographique, le substratum et/ou la roche-mère géologique, le type génétique de sol. Il s'agit bien entendu de peuplements de feuillus exclusivement; l'essence dominante y est très généralement le hêtre et le régime forestier celui de la futaie jardinée, l'âge de la réserve oscillant entre 100 et 150 ans. A l'exception de la station de Sainte-Marie qui représente un vieux taillis en reconversion vers la futaie, l'ensemble des stations se trouve à un état d'exploitation aussi voisin que possible.

Saint-Mard I, Bois de la Côte. Altitude 330 mètres.

- Hêtraie à flore du mull mésotrophe et du mull calcique.

- Pente faible en haut de versant, exposition sud-ouest.

- Manteau mixte et peu épais de limons soliflués et de terra fusca reposant sur le calcaire bajocien fragmenté. calcique.

- Sol brun calcique à mull eutrophe et rendzine brunifiée superficielle à mull

Saint-Mard II, Bois de la Côte. Altitude 390 mètres.

- Hêtraie mélangée à flore du mull mésotrophe.

- Plateau et début de pente faible vers l'ouest.

- Limons loessiques sur argile de décarbonatation du calcaire bajocien.

- Sol brun lessivé à mull mésotrophe.

Musson, Bois Pertot. Altitude 332 mètres.

- Hêtraie-chênaie à flore du mull mésotrophe.

- Plateau.

- Grès argilo-ferrugineux domérien (Macigno d'Aubange) avec contamination loessique en surface.

- Sol brun faiblement lessivé à mull mésotrophe.

Saint-Mard III, Bois de la Côte. Altitude 394 mètres.

- Hêtraie à flore du mull mésotrophe et oligotrophe.

- Vaste replat au sommet de la cuesta bajocienne.

- Loess sur matériau sablo-argileux de remplissage karstique.

- Sol lessivé acide à pseudogley sur paléosol glossique, à mull oligotrophe.

Udange, Bois d'Udange. Altitude 365 mètres.

- Hêtraie-chênaie à flore du mull mésotrophe et oligotrophe.

- Petit plateau.

- Grès ferrugineux domérien (Macigno de Messancy).

- Sol brun acide à mull oligotrophe.

Etalle, Bois d'Etalle. Altitude 345 mètres.

- Hêtraie à flore du mull acide.

- Plateau d'interfluve.

- Sables et sables argileux d'altération du grès calcaire d'Orval (Lotharingien).

- Sol brun acide légèrement cryptopodzolique, à mull-moder. 
Meix-devant-Virton, Bois de Meix-devant-Virton. Altitude 330 mètres.

- Hêtraie à flore du mull acide et du moder.

- Replat sommital et pente faible vers l'est.

- Sables et sables argileux d'altération du grès calcaire virtonien (Lotharingien).

- Sol brun cryptopodzolique à moder.

Gérouville, Bois de Nichansart. Altitude 325 mètres.

- Hêtraie à flore du moder.

- Petit plateau et pentes faibles avoisinantes.

- Sables et sables argileux d'altération du grès calcaire virtonien (Lotharingien).

- Sol brun cryptopodzolique à moder.

Sainte-Marie, Bois de Sainte-Marie. Altitude 335 mètres.

- Chênaie-hêtraie à flore du moder-mor.

- Plateau.

- Sable limoneux allogène avec cailloutis ferrugineux sur sable lotharingien.

- Sol ocre podzolique à dysmoder.

Châtillon, Bois de Châtillon. Altitude 370 mètres.

- Chênaie-hêtraie à flore du moder-mor.

- Plateau.

- Limon sableux allogène à charge de graviers ferrugineux sur argile d'altération des schistes d'Ethe (Carixien).

- Sol lessivé acide marmorisé à dysmoder.

Bellefontaine, Bois de Thonne-la-Long. Altitude 330 mètres.

- Chênaie-hêtraie à flore du moder-mor.

- Zone plane dans une topographie mollement vallonnée.

- Sables colluvionnés sur sables et sables argileux lotharingiens.

- Sol ocre podzolique à dysmoder.

\section{III. - Méthodes}

a. - Index et paramètres foliaires

Paramètres foliaires.

Dans les stations forestières étudiées divers paramètres foliaires ont été mesurés sur feuilles de litière récoltées au moment de la chute automnale (automne 1974). Les surfaces de feuilles sont mesurées à l'aide d'un photoplanimètre équipé de lentilles de Fresnel (Vanseveren, 1969). Sur un échantillon total d'environ 1000 feuilles d'une espèce sont déterminés les paramètres suivants : la surface moyenne (centimètre carré), le poids moyen de 100 feuilles (gramme) et la surface spécifique (centimètre carré/gramme). La surface spécifique des feuilles d'une espèce donnée est donc la surface, exprimée en centimètre carré, développée par unité de poids (gramme). La surface s'entend pour une simple face foliaire. 
Index foliaire (Leaf area index L.A.I.).

L'index foliaire des essences forestières dominantes et codominantes est mesuré en période automnale et les valeurs sont ensuite rapportées au stade de développement foliaire maximum, c'est-à-dire à la période de pleine feuillaison. L'index est calculé à partir des valeurs de surface spécifique mesurées par photoplanimétrie et des masses de litière (récoltées lors des retombées automnales) et exprimées en kilogramme/hectare. La masse de litière foliaire est déterminée en relevant 10 placeaux de 1 mètre carré répartis au hasard au sein de chaque station. L'index foliaire ainsi obtenu est exprimé en mètre carré de surface de fevilles par mètre carré de sol. En tenant compte du coefficient de rétraction des limbes foliaires frais, on peut calculer un index en surface de feuilles fraîches (Vanseveren, 1969).

Dans un travail antérieur au cours duquel ont été analysées les variations annuelles et saisonnières de l'index foliaire de chênaies et de hêtraies, ceci pour une séquence de 9 années successives, l'un d'entre nous a pu montrer que la variabilité attachée à l'index foliaire n'est que de l'ordre de 10 p. 100 . On peut donc admettre que l'incidence des fluctuations annuelles sur la valeur mesurée du L.A.I. est faible. II est également apparu que l'index foliaire d'une forêt dépend essentiellement de la surface spécifique des limbes et, dans une bien moindre mesure, de la biomasse foliaire élaborée par le peuplement ; cette biomasse est, par ailleurs, relativement constante d'une année à l'autre.

\section{b. - Caractéristiques édaphiques}

Pour chaque station un profil complet a fait l'objet d'analyses et le type génétique de sol a été déterminé avec précision. Nous n'avons toutefois pas jugé utile de joindre au présent article les fiches signalétiques de chaque profil et nous ne mentionnons au tableau II que les valeurs relatives aux horizons hémorganiques $A_{1}$. C'est d'ailleurs l'horizon humifère que nous avons choisi dans ce travail comme caractéristique édaphique stationnelle ; à la suite de nombreux travaux, on sait, en effet, que ce sont dans une très large mesure les horizons humifères qui intègrent le mieux les conditions stationnelles en milieu forestier (Duchaufour 1970, Manil et al. 1963, Duvigneaud et Froment 1969, Decourt et Le Tacon 1970, etc.), d'où le choix fréquent du type d'humus comme facteur synthétique discriminant au niveau stationnel (Garbaye et al., 1970).

Les analyses de caractérisation ont été effectuées par les méthodes conventionnelles suivantes:

- cations échangeables : dosage après percolation à l'acétate d'ammonium neutre normal par photométrie de flamme $(\mathrm{Ca}, \mathrm{K})$ ou absorption atomique $(\mathrm{Mg})$. $\mathrm{NaCl} 0,5 \mathrm{~N}$.

- C.E.C. : dosege en retour de l'ammonium déplacé par une solution de

- carbone total : dosage direct au Carmograph 12 WOSTHOFF *.

— azote total : méthode de Kjeldahl.

* Les dosages de carbone ont été effectués avec l'aide de Mme B. Gérard au Laboratoire de Pédologie de la Faculté des Sciences de l'Université de Nancy I. 
- activité minéralisatrice de l'azote : technique classique d'incubation in vitro, à $28^{\circ} \mathrm{C}$ pendant 6 semaines (disponibilité potentielle); le dosage des formes minérales d'azote a été effectué soit par la méthode de Bremner (extraction par $\mathrm{CaCl}_{2} \mathrm{~N}$ et dosage titrimétrique après entraînement à la vapeur en présence de $\mathrm{MgO}$ et de $\mathrm{MgO}+$ alliage de Devarda), soit par colorimétrie à l'analyseur TECHNICON après extraction au KCL $N * *$. Dix incubations échelonnées sur l'année sont effectuées pour chaque horizon humifère, en sorte que la valeur mentionnée correspond à un taux de minéralisation moyen.

\section{IV. - Résultats}

\section{a. - Index foliaire et paramètres foliaires}

Le tableau 1 regroupe l'ensemble des facteurs biologiques choisis pour caractériser et comparer les peuplements étudiés. Ces derniers sont hiérarchisés par ordre de richesse édaphique décroissante. Le hêtre (Fagus sylvatica) est présent dans les 11 stations forestières, dont il constitue d'ailleurs souvent l'essence dominante et les paramètres foliaires mentionnés se rapportent à cette espèce. II s'agit de paramètres tels que la surface spécifique des feuilles, la surface moyenne, le poids moyen, l'index foliaire du peuplement et la masse de litière.

Avant d'examiner les relations qui lient paramètres foliaires et caractéristiques édaphiques stationnelles, il convient de voir dans quelle mesure les paramètres pris en considération sont significativement différents entre les divers peuplements étudiés.

- Retombées automnales de litière.

La masse de litière foliaire recueillie dans les 11 stations varie de 1871 à 3166 kilogrammes/hectare, chiffres dont l'ordre de grandeur est comparable à ceux obtenus pour la Lorraine française par Aussenac (1969) et Toutain et Duchaufour (1970). En traitant les valeurs de litière (kilogramme/hectare) par analyse de la variance à un facteur de contrôle, on constate que la variation de la masse de litière entre les peuplements est très nettement supérieure à la variation de ce paramètre au sein de chaque forêt ( $F$ calculé $5,45 F>$ table 3,30 à $P 99,9 p$. 100). Il y a donc des différences très hautement significatives entre les quantités de litière foliaire récoltées dans les 11 stations d'étude (automne 1974). La variabilité attachée aux moyennes des masses de litière recueillies oscille en règle générale autour de 16 p. 100 .

Des valeurs consignées dans le tableau 1 , il ressort que les associations forestières les plus riches (stations à mull) ne sont pas nécessairement celles qui produisent la plus grande masse de feuilles.

- Index foliaire.

Les valeurs de l'index foliaire dans les 11 forêts étudiées oscillent entre 3,02 et 6,98 unités (tabl. 1). On peut noter, dès à présent, que les valeurs du L.A.I., bien que

** S. Martin a réalisé à la Fondation Universitaire Luxembourgeoise les dosages à I'analyseur TECHNICON, selon un protocole mis au point par P. Warzée. 
Index foliaire, retombées de litière et paramètres foliaires dans 11 peuplements forestiers de Lorraine belge Leaf area index, leaf fall and leaf parameters in 11 forest stands of south Belgium

\begin{tabular}{|c|c|c|c|c|c|c|c|c|c|}
\hline \multirow[b]{2}{*}{ Stations } & \multirow{2}{*}{$\begin{array}{l}\text { Retom- } \\
\text { bées } \\
\text { foliaires } \\
\mathrm{kg} / \mathrm{ha}\end{array}$} & \multirow{2}{*}{$\begin{array}{l}\text { Litière } \\
\text { variabi- } \\
\text { lité } \\
\text { p. } 100\end{array}$} & \multirow[b]{2}{*}{$\begin{array}{l}\text { Index } \\
\text { foliaire }\end{array}$} & \multicolumn{6}{|c|}{ Paramètres foliaires de Fagus sylvatica } \\
\hline & & & & $\begin{array}{c}\text { Surface } \\
\text { spécifique } \\
\mathrm{cm}^{2} / \mathrm{g}\end{array}$ & $\begin{array}{l}\begin{array}{c}\text { Variabi- } \\
\text { lité }\end{array} \\
\text { p. } 100\end{array}$ & $\begin{array}{l}\text { Surface } \\
\text { moyenne } \\
\mathrm{cm}^{2}\end{array}$ & $\begin{array}{l}\begin{array}{c}\text { Variabi- } \\
\text { lité } \\
\text { p. } 100\end{array}\end{array}$ & $\begin{array}{l}\text { Poids } \\
\text { moyen } \\
100 \mathrm{f.g} .\end{array}$ & $\begin{array}{c}\text { Variabi- } \\
\text { lifé } \\
\text { p. } 100\end{array}$ \\
\hline 1. Hêtraie à mull calcique ...... & 2614 & 18,0 & 5,93 & 213,0 & 2,48 & 16,6 & 7,83 & 7,8 & 6,64 \\
\hline 2. Hêtraie à mull mésotrophe ... & 2824 & 16,7 & 5,61 & 195,1 & 2,71 & 18,7 & 6,95 & 9,6 & 5,40 \\
\hline $\begin{array}{l}\text { 3. Hêtraie-chênaie à mull méso- } \\
\text { trophe } \ldots \ldots \ldots \ldots \ldots \ldots \ldots\end{array}$ & 3166 & 14,9 & 6,98 & 210,0 & 2,52 & 14,0 & 9,29 & 6,7 & 7,73 \\
\hline 4. Hêtraie à mull oligotrophe ... & 2788 & 16,9 & 5,96 & 205,3 & 2,58 & 17,6 & 7,39 & 8,6 & 6,02 \\
\hline trophe $\ldots \ldots \ldots \ldots \ldots \ldots$ & 2620 & 18,0 & 5,44 & 243,5 & 2,17 & 18,4 & 7,07 & 7,5 & 6,91 \\
\hline 6. Hêtraie à mull moder $\ldots \ldots \ldots$ & 2648 & 17,8 & 5,40 & 215,9 & 2,45 & 15,2 & 8,55 & 7,0 & 7,40 \\
\hline 7. Hêtraie à moder...$\ldots \ldots \ldots$ & 2382 & 19,8 & 5,33 & 211,2 & 2,50 & 15,3 & 8,50 & 7,2 & 7,19 \\
\hline 8. Hêtraie à moder $\ldots \ldots \ldots \ldots$. & 2828 & 16,7 & 6,45 & 221,2 & 2,39 & 17,0 & 7,65 & 7,7 & 6,72 \\
\hline 9. Chênaie-hêtraie à moder-mor & 2522 & 18,7 & 4,95 & 225,5 & 2,35 & 17,7 & 7,34 & 7,8 & 6,64 \\
\hline 10. Chênaie-hêtraie à moder-mor & 3060 & 15,4 & 5,98 & 223,2 & 2,37 & 14,4 & 9,02 & 6,5 & 7,97 \\
\hline 11. Chênaie (hêtraie) à moder-mor & 1871 & 25,2 & 3,02 & 232,4 & 2,28 & 20,4 & 6,37 & 8,8 & 5.89 \\
\hline
\end{tabular}


fort variables, ne peuvent être mises en relation avec la variation des facteurs stationnels, des index peu élevés ayant été mesurés aussi bien dans des forêts acidophiles que mésophiles.

Les valeurs d'index foliaire ici obtenues s'insèrent, en fait, dans l'intervalle des valeurs habituellement mentionnées dans la littérature pour les peuplements feuillus (chênaies et hêtraies) de la zone des forêts caducifoliées tempérées (L.A.I. de 4 à 9 unités) (Vanseveren, 1976).

II semble donc impossible de pouvoir utiliser l'index foliaire comme éventuelle caractéristique stationnelle d'un peuplement de feuillus.

\section{- Paramètres foliaires du hêtre.}

L'analyse de la variance appliquée aux valeurs des paramètres foliaires de Fagus (surface spécifique, surface moyenne et poids moyen), fournit les résultats suivant (ces paramètres s'appliquent à des feuilles de litière fraîche) :

$\begin{array}{ll}\text { surface spécifique : F 52,40*** } \\ \text { surface moyenne } & \text { : F } 16,60 * * * \\ \text { poids moyen } & \text { : F } 28,69 * * *\end{array}$

II ressort de ces chiffres que les 3 paramètres foliaires envisagés marquent des différences très hautement significatives entre les 11 types de peuplement. L'amplitude de variation de la surface spécifique dans les diverses phytocénoses forestières étudiées varie de 211,2 à 243,5 centimètres carrés/gramme. La surface moyenne ef le poids moyen de 100 feuilles de hêtres (feuilles de litière) fluctuent respectivement de 14,0 à 20,5 centimètres carrés et de 6,5 à 9,6 grammes. Les valeurs moyennes des paramètres foliaires sont affectées d'une variabilité faible : 2,5 p. 100 pour la surface spécifique et 7 p. 100 pour la surface moyenne et le poids moyen des feuilles.

\section{b) Caractéristiques des sols}

Les caractéristiques chimiques des horizons humifères des 11 stations sont consignées dans le tableau II. II ressort clairement de ces chiffres que la gamme de richesse édaphique sélectionnée est aussi large que possible, puisqu'elle recouvre la séquence presque complète des types d'humus dont les extrêmes sont ici représentés, d'une part, par des sols à humus calcique saturé ef, d'autre part, par des humus de type moder fortement désaturés et très acides, les termes moyens étant assurés par des mull mésotrophes et oligotrophes.

La disponibilité potentielle en azote minéral et surtout la qualité de la minéralisation azotée (rapport nitrification/ammonification) sont également le reflet assez fidèle de ce gradient de fertilité. Rappelons que l'azote en tant que facteur stationnel est un indice particulièrement précieux comme l'ont montré notamment les travaux classiques d'Ellenberg (1964), de Lemée (1968) et de Zöttl (1960) ; c'est d'ailleurs à la suite des travaux de Zöttl sur les peuplements résineux de Bavière que divers auteurs ont utilisé les caractéristiques de la minéralisation azotée comme critère de fertilité édaphique au niveau stationnel (Le Tacon 1972, Van Praag et Weissen 1973). 
Principales caractéristiques analytiques des horizons humifères des onze stations inventoriées

Les valeurs de $\mathrm{Ca}, \mathrm{Mg}$ et $\mathrm{K}$ échangeables, de leur somme (S) et de la capacité d'échange cationique (T) sont données en $\mathrm{m}$. e. $100 \mathrm{~g}$ t. s., le taux de saturation $(\mathrm{S} / \mathrm{T})$ en $\%$, les taux de $\mathrm{M}$. O. (Matière organique), de carbone $(\mathrm{C})$, ef d'azote $(\mathrm{N})$ en \% également; les taux de nitrification et de minéralisation nette de l'azote sont exprimés en \% (minéralisation potentielle in vitro); la production d'azote minéral et de sa forme nitrique est également mentionnée en $\mathrm{kg} / \mathrm{ha}\left(\mathrm{Nm}\right.$ ef $\left.\mathrm{N}_{\mathrm{NO}_{3}}\right)$.

Basic analytical data for humus samples from eleven forest stands studied in south Belgium (Lorraine)

Exchangeable cations ( $\mathrm{Ca}, \mathrm{Mg}, \mathrm{K})$, their sum (S) and the cation exchange capacity (C. E. C. $=\mathrm{T}$ ) are given in $\mathrm{m}$. e. $/ 100 \mathrm{~g}$ dried soil. Saturation rate $(\mathrm{S} / \mathrm{T})$, nitrification ratio and nitrogen net mineralization rate are given in \% dry weight; net production of mineral nitrogen $\left(\mathrm{Nm}^{2}\right)$ and the nitric form $\left(\mathrm{N}_{\mathrm{NO}}\right)$
are also given in $\mathrm{kg} / \mathrm{ha}$.

\begin{tabular}{|c|c|c|c|c|c|c|c|c|c|c|c|c|c|c|c|c|}
\hline Station & Type d'humus & $\mathrm{pH}$ & $\mathrm{Ca}$ & $\mathrm{Mg}$ & K & $\mathrm{S}$ & $\mathrm{T}$ & $S / T$ & M. O. & C & $N$ & $\mathrm{C} / \mathrm{N}$ & $\mathrm{N}_{\mathrm{NO}_{3}} / \mathrm{Nm}$ & $\mathrm{Nm} / \mathrm{Nt}$ & $\mathrm{N}_{\mathrm{NO}_{3}}$ & $\mathrm{Nm}$ \\
\hline Saint-Mard I . & Mull calcique & 6,6 & - & 2,29 & 0,85 & - & 41,5 & sat & 10,5 & 5,26 & 0,35 & 15,0 & 100 & 1,1 & 38 & 38 \\
\hline Saint-Mard II & Mull mésotrophe & 5,4 & 8,00 & 1,19 & 0,35 & 9,54 & 17,5 & 54,5 & 7,3 & 3,63 & 0,27 & 13,2 & 50,6 & 3,6 & 47 & 88 \\
\hline Musson & Mull mésotrophe & 5,2 & 13,50 & 1,46 & 1,02 & 15,98 & 24,8 & 64,4 & 13,2 & 6,58 & 0,45 & 14,7 & 45,4 & 4,3 & 85 & 180 \\
\hline Saint-Mard III . & Mull oligotrophe & 4,7 & 7,38 & 0,98 & 0,45 & 8,81 & 29,0 & 30,4 & 15,0 & 7,50 & 0,51 & 14,8 & 68,6 & 6,3 & 85 & 123 \\
\hline Udange & Mull oligotrophe & 4,8 & 8,75 & 1,02 & 0,77 & 10,54 & 28,5 & 36,9 & 17,3 & 8,65 & 0,54 & 16,0 & 20,7 & 4,5 & 36 & 174 \\
\hline Etalle & Mull-Moder & 4,5 & 0,85 & 0,29 & 0,14 & 1,27 & 5,7 & 22,4 & 3,7 & 1,84 & 0,14 & 13,3 & 25,9 & 8,0 & 31 & 122 \\
\hline Meix-dt-Virton & Moder & 4,1 & 1,46 & 0,42 & 0,21 & 2,09 & 9,3 & 22,5 & 5,3 & 2,62 & 20,16 & 16,7 & 40,4 & 4,5 & 14 & 39 \\
\hline Gerouville. & Moder & 4,1 & 1,18 & 0,26 & 0,23 & 1,67 & 9,1 & 18,4 & 8,2 & 4,11 & 0,24 & 16,9 & 2,7 & 7,2 & 2 & 67 \\
\hline Ste Marie & Dysmoder & 4,5 & 2,00 & 0,79 & 0,58 & 3,31 & 21,8 & 315,2 & 21,1 & 10,54 & 40,60 & 17,6 & 0 & 2,7 & 0 & 32 \\
\hline Chatillon & Dysmoder & 4,5 & 0,94 & 0,44 & 0,31 & 1,69 & 15,0 & 11,3 & 11,8 & 5,92 & 20,33 & 17,8 & 7,3 & 3,8 & 7 & 72 \\
\hline Bellefontaine & Dysmoder & 3,9 & 0,57 & 0,28 & 0,20 & 1,05 & 9,0 & 11,7 & 13,7 & 6,86 & 60,38 & 18,0 & 1,0 & 3,6 & $\mathrm{Tr}$ & 18 \\
\hline
\end{tabular}




\section{V. - Relations entre les paramètres foliaires et les caractéristiques édaphiques}

\section{A. - Relation générale avec le type d'humus}

Une comparaison entre paramètres foliaires du hêtre et indice de richesse stationnelle (intégrée globalement par le type d'humus) ne fait apparaître une relation qu'arec un seul paramètre foliaire, la surface spécifique ; la surface moyenne ou le poids moyen des feuilles de Fagus sont quant à eux des paramètres absolument indépendants de la richesse du milieu. Nous avons également signalé précédemment qu'aucun lien ne peut être décelé entre l'index foliaire des peuplements et les caractéristiques stationnelles.

Les valeurs des surfaces spécifiques se différencient plus ou moins nettement en 2 ordres de grandeur selon la qualité édaphique des stations : les stations « riches» du mull calcique et du mull mésotrophe correspondent à des valeurs de surface spécifique oscillant de 195 à 213 centimètres carrés/gramme; les autres stations, du mull acide au moder-mor, présentent des surfaces spécifiques qui dépassent 211 centimètres carrés/gramme, avec une nette tendance à augmenter à mesure que la richesse du sol diminue. Ceci corrobore une observation similaire qui avait été faite pour d'autres types forestiers de Haute Belgique (Vanseveren, 1976).

\section{B. - Relations sélectives en fonction de caractéristiques édaphiques précises}

Afin d'essayer de préciser les relations paramètres foliaires-caractéristiques édaphiques stationnelles et de déceler éventuellement l'incidence prépondérante de l'un ou l'autre facteur pédologique, nous avons soumis à l'analyse statistique les valeurs des paramètres foliaires et de quelques indices de caractérisation des sols généralement considérés comme discriminants au point de vue écosystémique (indice de richesse minérale : somme des cations échangeables et taux de saturation ; indice d'activité biologique et de richesse azotée des humus : rapport $\mathrm{C} / \mathrm{N}$; indice de disponibilité en azote et de qualité de minéralisation : taux de minéralisation potentiel de l'azote et taux de nitrification).

\section{TABLEAU 3}

Coefficients de corrélation linéaire calculés pour la relation paramètres foliaires - caractéristiques édaphiques dans 11 peuplements forestiers de Lorraine belge

Linear correlation coefficients calculated between leaf parameters and edaphic parameters in 11 foreststands of south Belgium

\begin{tabular}{|c|c|c|c|}
\hline & Surface spécifique & Surface moyenne & Poids moyen \\
\hline $\begin{array}{l}\mathrm{S} \text { m. e. } / 100 \mathrm{~g} \ldots \ldots \ldots \ldots \ldots \\
\mathrm{S} / \mathrm{T} . \ldots \ldots \ldots \ldots \ldots \ldots \\
\mathrm{C} / \mathrm{N} \ldots \ldots \ldots \ldots \ldots \ldots \\
\mathrm{N} . \mathrm{NO}_{3} \mathrm{~kg} / \mathrm{ha} \ldots \ldots \ldots \ldots \ldots \ldots \\
\mathrm{N} . \mathrm{NO}_{3} / \mathrm{Nm} \ldots \ldots \ldots \ldots \ldots \ldots \\
\mathrm{Nm} / \mathrm{Nt} \ldots \ldots \ldots \ldots \ldots \ldots\end{array}$ & $\begin{array}{l}-0,242 \mathrm{NS} \\
-0,405 \mathrm{NS} \\
0,674 * \\
-0,490 \mathrm{NS} \\
-0,709 * * \\
-0,074 \mathrm{NS}\end{array}$ & $\begin{array}{r}-0,136 \mathrm{NS} \\
-0,166 \mathrm{NS} \\
0,332 \mathrm{NS} \\
-0,203 \mathrm{NS} \\
-0,160 \mathrm{NS} \\
-0,210 \mathrm{NS}\end{array}$ & $\begin{array}{r}0 \mathrm{NS} \\
0,063 \mathrm{NS} \\
-0,041 \mathrm{NS} \\
0,079 \mathrm{NS} \\
0,273 \mathrm{NS} \\
-0,170 \mathrm{NS}\end{array}$ \\
\hline
\end{tabular}



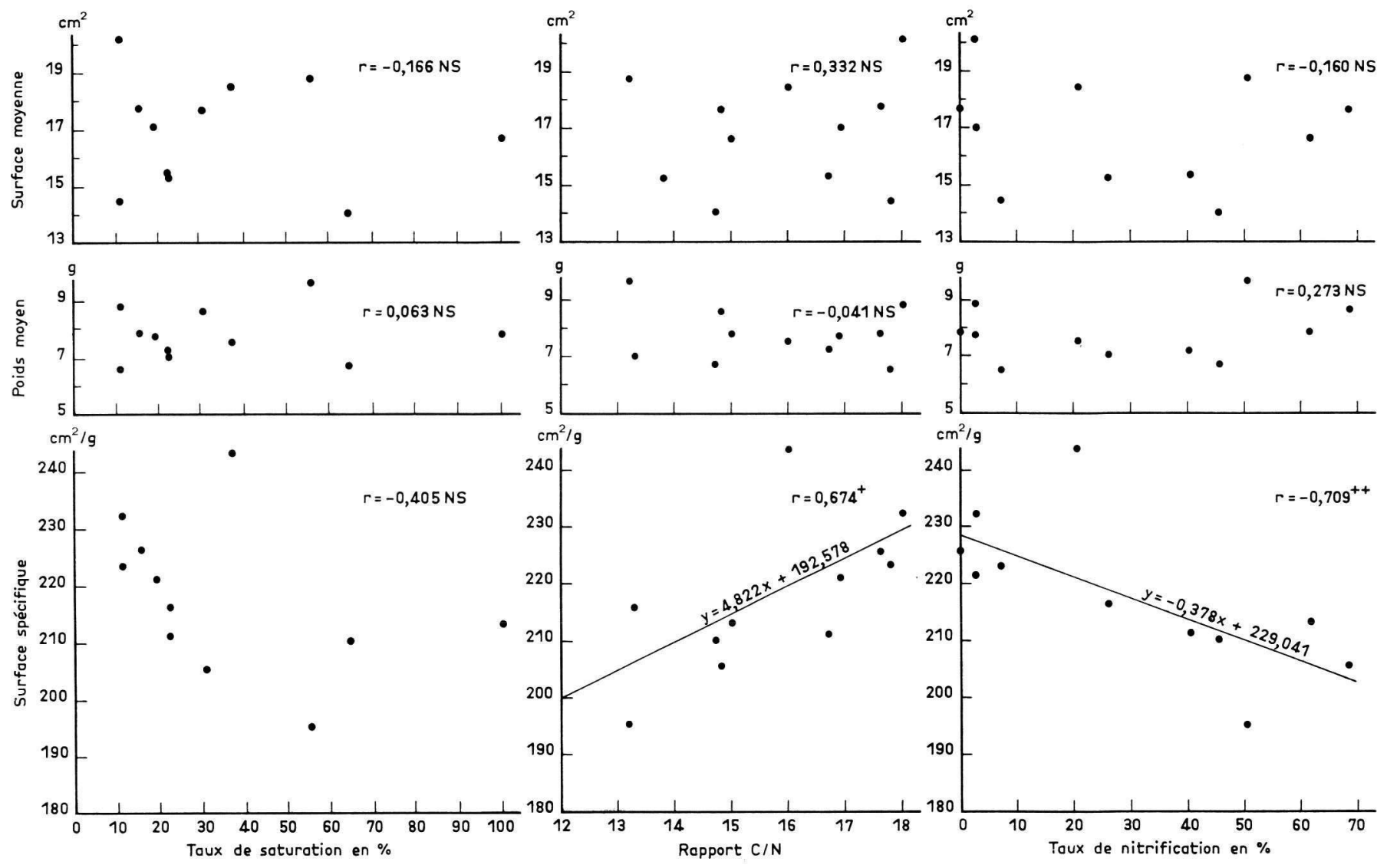

FIG. 2. - Relation entre paramètres foliaires et caractéristiques des horizons humifères. Relation between foliar parameters and edaphic characteristics of humus layers. 
Les résultats obtenus sont rassemblés dans le tableau III et certaines relations sont figurées graphiquement à la figure 2.

On constate que la plupart des corrélations sont non significatives, exception faite du paramètre foliaire surface spécifique pour lequel deux corrélations s'avèrent statistiquement significatives, tout au moins dans les limites de notre échantillonnage $(n=11)$. Il apparaît en effet que la surface spécifique des feuilles de Fagus est liée négativement, et de façon hautement significative au seuil de 99 p. $100(r=0,709 * *)$, au taux de nitrification des humus (par voie de conséquence, la relation est positive avec le taux d'ammonification); la relation avec le rapport $C / N$ est positive mais moins bonne $(r=0,674 *)$. Il est étonnant de constater que la corrélation devient non significative lorsque l'on prend en considération les valeurs d'azote nitrique rapportées en kilogramme/hectare, mode d'expression généralement considéré comme plus écologique.

\section{VI. - Conclusions}

A l'issue d'une comparaison des paramètres foliaires de Fagus sylvatica dans 11 peuplements feuillus de Lorraine belge croissant sur une gamme variée de sols, des rendzines brunifiées aux sols ocres podzoliques, et des caractéristiques édaphiques des horizons humifères de ces sols, on a pu mettre en évidence une relation entre la surface spécifique des feuilles de hêtre et le pouvoir nitrifiant des horizons humifères. Au fur et à mesure que décroissent les potentialités nitrificatrices des humus, on constate que la surface spécifique des feuilles de Fagus s'accroît. Cette corrélation est vérifiée statistiquement dans les limites de notre échantillonnage $(r=-0,709 * *$ pour $n=11)$. Une corrélation positive entre ce même paramètre foliaire et le rapport $\mathrm{C} / \mathrm{N}$ a également pu être décelée $(r=0,674 *)$. Rappelons qu'une observation parallèle avait déjà été faite dans d'autres peuplements forestiers de Haute Belgique où l'un d'entre nous a pu montrer que la surface spécifique des feuilles de hêtre a tendance à augmenter à mesure que diminue la qualité édaphique des sites (Vanseveren, 1976). Au vu de ces premiers résultats, il semblerait donc que les modifications des conditions édaphiques soient à même d'influencer sensiblement non seulement la productivité des essences forestières comme cela a été démontré à de multiples reprises, mais aussi des caractéristiques structurelles de ces essences, telles que certains paramètres foliaires.

La plupart des corrélations testées entre paramètres foliaires, index foliaire et variables édaphiques se sont cependant révélées non significatives. La confrontation de données relatives à des caractéristiques des peuplements (le choix s'étant porté ici sur divers paramètres foliaires et l'index foliaire) et de déterminations pédologiques synthétiques ou sélectives, s'avère en définitive assez décevante par les résultats limités qu'elle fournit. On se trouve par ailleurs contraint de travailler, et ceci pour des raisons matérielles, sur un échantillonnage stationnel relativement restreint, ce qui limite inévitablement la portée des résultats obtenus. Mais une première approche telle qu'elle a été tentée dans le présent travail permet cependant de sélectionner parmi un ensemble de facteurs édaphiques celui qui semble influer de façon prépondérante sur l'une ou l'autre des caractéristiques biologiques du peuplement que l'on a choisi de mesurer. L'étape suivante consiste alors à inventorier un plus grand nombre 
de stations pour confirmer ou infirmer la corrélation qui a pu être mise en évidence dans la première phase de l'étude, cette augmentation du nombre de points de mesure étant rendue matériellement possible par la réduction des variables à mesurer. Un tel travail est actuellement poursuivi dans plus de 30 sites forestiers de Lorraine belge.

Reçu pour publication en février 1977.

\author{
Summary \\ Leaf area index, leaf parameters and edaphic parameters \\ in some forest stands of south Belgium
}

A comparison between some characteristics of deciduous forests (foliar parameters and leaf area index) and edaphic parameters (i. e. humus type, $\mathrm{C} / \mathrm{N}$ ratio, nitrogen mineralization of the humus layers) has been made, during the same year, in eleven forest stands in south Belgium (Lorraine belge), especially beech forests, growing on various types of soils. Statistical correlations has been founded between some charasteristics of the soils and the specific surface of beech foliage. This foliar parameter tends to increase as the richness of the soil decreases.

\title{
Références bibliographiques
}

AUSSENAC G., 1969. Production de litière dans divers peuplements forestiers de l'Est de la France. Oecol. Plant., 4, 3, 225-235.

COÛTEAUX M., 1969. Recherches écologiques sur les forêts de Gaume. Bull. Jard. Bot. Nat. Belg., 39, 3, 227-311 et 39, 4, 313-343.

DAGNELIE P., HUBERTY J.-P., NOIRFALISE A., 1960. Recherches sur la productivité des hêtraies des macignos et des marnes du Bas-Luxembourg. Bull. Inst. Agr. Stat. Rech. Gembloux, 28, 1, 18-32.

DECOURT N., LE TACON F., 1970. L'Epicéa commun (Picea excelsa) sur les plareaux calcaires de l'est de la France. Essai de prévision de la production à l'aide de déterminations pédologiques simples. Ann. Sci. forest., 27, 3, 255-286.

DUCHAUFOUR Ph., 1970. Précis de Pédologie. Masson et Cie, 481 p.

DUVIGNEAUD P., 1960. Carte écologique des groupements potentiels de Gaume. 1/40 000e. Ministère des Travaux Publics et de la Reconstruction. Administration centrale de l'Urbanisme. Enquête régionale du Bas-Luxembourg, C.E.B.L.

DUVIGNEAUD P., FROMENT A., 1969. Recherches sur l'Ecosystème forêt. Série E. Contrib. no 5 : Eléments biogènes de l'édaphotope et phytocénose forestière. Bull. Inst. r. Sci. nat. Belg., 45, 25, 1-48.

ELLENBERG H., 1964. Stickstoff als Standortsfactor. Ber. deutsch. Bot. Ges., 77, 1, 82-92.

GARBAYE J., LEROY Ph., LE TACON F., LEVY G., 1970. Réflexions sur une méthode d'étude des relations entre facteurs écologiques et caractéristiques des peuplements. Ann. Sci. forest., 27, 3, 303-321.

LEMEE G., 1967. Investigations sur la minéralisation de l'azote et son évolution annuelle dans les humus forestiers in situ. Oecol. Plant., 2, 4, 285-323.

LE TACON F., 1972. Disponibilité de l'azote nitrique et ammoniacal de certains sols de l'est de la France. Ann. Sci. forest., 30, 2, 183-203.

MANIL G., DELECOUR F., FORGET G., EL ATTAR A., 1963. L'humus, facteur de station dans les hêtraies acidophiles de Belgique. Bull. Inst. Agron. Stat. Rech. Gembloux, 31, 1 et 2, 1-114.

SCHNOCK G., 1967. Les forêts actuelles du Sud-Est belge. Carłographie, inventaire et répartition des principaux types de peuplement. Bull. Soc. Roy. Forest. Belg., 3-58.

SOUGNEZ N., 1967. Les forêts de la Lorraine belge. Etude phytosociologique. Les Presses Agronomiques de Gembloux, 114 p.

STEFFENS R., 1971. Les sols de la Lorraine belge. Pédologie, Mémoire 4, 392 p.

TANGHE M., 1967. Les groupes écologiques forestiers de la Gaume. Lejeunia, N. S., 43, 1-64.

TOUTAIN F., DUCHAUFOUR Ph., 1970. Etude comparée des bilans biologiques de certains sols de hêtraie. Ann. Sci. forest., 27, 1, 39-61. 
VANSEVEREN J. P., 1969. Recherches sur l'écosystème forêt. Série B : La chênaie mélangée calcicole de Virelles-Blaimont. L'index foliaire et sa mesure par photoplanimétrie. Bull. Soc. Roy. Bot. Belg., 102, 373-385.

VANSEVEREN J. P., 1976. Facteurs extrinsèques et intrinsèques de productivité dans quelques biogéocénoses forestières de haute Belgique. Thèse, Université Libre de Bruxelles, $259 \mathrm{p}$.

VAN PRAAG H., WEISSEN F., 1973. Elements of a functionnal definition of oligotroph humus based

ZÖTTL H., 1960 . Methodische Untersuchungen zur Bestimmung der Mineralstickstoffnachlieferung des Waldbodens. Forst. Cbl., 79, 3-4, 72-88.

ZÖTTL H., 1960. Die Mineralstickstoffanlieferung in Fichten und Kiefernbeständen Bayerns. Forstw. Cbl., 79, 7-8, 221-236. 